\title{
Generating a transgenic mouse line stably expressing human MHC surface antigen from a HAC carrying multiple genomic BACs
}

\author{
Yoshinori Hasegawa • Tomoyuki Ishikura • Takanori Hasegawa • Takashi Watanabe • \\ Junpei Suzuki • Manabu Nakayama • Yoshiaki Okamura • Tuneko Okazaki • \\ Haruhiko Koseki • Osamu Ohara • Masashi Ikeno • Hiroshi Masumoto
}

Received: 7 July 2014 / Revised: 24 September 2014 / Accepted: 24 September 2014 / Published online: 12 October 2014

(C) The Author(s) 2014. This article is published with open access at Springerlink.com

\begin{abstract}
The human artificial chromosome (HAC) vector is a promising tool to improve the problematic suppression and position effects of transgene expression frequently seen in transgenic cells and animals produced by conventional plasmid or viral vectors. We generated transgenic mice maintaining a single HAC vector carrying two genomic bacterial artificial chromosomes (BACs) from human HLA-DR loci (DRA and DRB1). Both transgenes on the HAC in transgenic mice exhibited tissue-specific expression in kidney, liver, lung, spleen, lymph node, bone marrow, and thymus cells in RT-PCR analysis. Stable functional expression of a cell surface HLA-DR marker from both transgenes, DRA and DRB1 on the HAC, was detected by flow cytometric analysis of splenocytes and maintained through at least eight filial generations. These results indicate that the de novo HAC system can allow us to manipulate multiple BAC transgenes with
\end{abstract}

Electronic supplementary material The online version of this article (doi:10.1007/s00412-014-0488-3) contains supplementary material, which is available to authorized users.

Y. Hasegawa $\cdot$ Y. Okamura $\cdot$ H. Masumoto $(\bowtie)$

Laboratory of Cell Engineering, Department of Frontier Research,

Kazusa DNA Research Institute, 2-6-7 Kazusa-Kamatari, Kisarazu, Chiba 292-0818, Japan

e-mail: masumoto@kazusa.or.jp

Y. Hasegawa $\cdot$ J. Suzuki $\cdot$ M. Nakayama $\cdot$ O. Ohara

Department of Technology Development, Kazusa DNA Research

Institute, 2-6-7 Kazusa-Kamatari, Kisarazu, Chiba 292-0818, Japan

T. Ishikura $\cdot$ T. Hasegawa $\cdot$ T. Watanabe $\cdot$ H. Koseki $\cdot$ O. Ohara RIKEN Center for Integrative Medical Sciences (IMS-RCAI), 1-7-22 Suehiro-cho Tsurumi-ku, Yokohama, Kanagawa 230-0045, Japan

T. Okazaki $\cdot$ M. Ikeno

Chromo Research Inc., 1212 Shihongi, Midori-ku, Nagoya,

Aichi 458-0039, Japan coordinated expression as a surface antigen through the generation of transgenic animals.

\section{Introduction}

Transgenic animals have provided tools for investigating many biological problems. Genomic fragments cloned by bacterial artificial chromosomes (BACs) have been utilized to generate transgenic animals when tissue-specific or temporally controlled expression of transgenes is desired. Due to the large insert capacity $(\sim 350 \mathrm{~kb})$ of a BAC vector, the genomic fragments can often possess the complete promoters and control elements of the gene of interest (Asami et al. 2011). In addition, BAC transgenes seem to be more resistant to position effects than smaller transgenes, such as artificial expression cassettes with complementary DNA (cDNA) (Gong et al. 2003). Typically, BAC transgenic mice are generated by microinjection of the BAC DNA into the pronucleus of fertilized mouse eggs (Vintersten et al. 2008). However, in principle, this method causes random integration (nonspecific insertion) of BAC DNAs into the mouse genome, and the number of insertion copies is variable. Increased copy number of a BAC transgene correlates with increased expression of the BAC transgene (Chandler et al. 2007). When investigating the cooperation of two transgenes in a transgenic mouse, generally two characterized transgenic mouse lines are crossed, but this is a time-consuming method and maintaining an appropriate level of gene expression is difficult.

A de novo human artificial chromosome (HAC) was constructed with naked human centromeric repetitive DNA (Harrington et al. 1997; Ikeno et al. 1998) and a HAC vector system developed in which one copy of a DNA fragment can be handled by Cre/lox insertion and transferred into a variety 
of vertebrate cell lines (Ikeno et al. 2009; Iida et al. 2010). A $\mathrm{HAC}$ is an episomal vector that can harbor a large DNA and is exploitable for generating transgenic animals using embryonic stem (ES) cell technology (Kazuki and Oshimura 2011; Ikeno et al. 2012). Thus, the HAC system can avoid the copy number problem and/or position effects caused by nonspecific insertion of the BAC transgene. The HAC vector is expected to be available for the production of transgenic mice carrying two or more single-copy genes with a large control region over tens of kilobases. Recently, a transgenic mouse harboring a single copy of a HAC, termed a trans-minichromosomal (TMC) mouse, carrying three continuous noncorrelated genes from the human genome was generated by inserting a single BAC DNA from chromosome 21 (Miyamoto et al. 2014). However, whether two or multiple independent $\mathrm{BAC}$ transgenes can be gathered onto a single $\mathrm{HAC}$ and cooperatively function in a transgenic mouse has not been investigated.

Here, we describe a transgenic mouse using a HAC vector carrying two single-copy human HLA-DR genomic genes. HLA-DR is a major histocompatibility complex (MHC) class II cell surface receptor consisting of an $\alpha \beta$ heterodimer. We introduced a DR $\alpha$-chain (HLA-DRA locus) and DR $\beta$-chain (HLA-DRB $1 * 0405$ locus) into a single HAC vector (HLAHAC). In transgenic mice harboring HLA-HAC (carrying HLA-DRA and DRB1 genes), tissue-specific expression of human MHC class II cell surface receptor in spleen cells was detected by flow cytometric analysis through at least eight filial generations.

\section{Materials and methods}

\section{Cell culture}

Chinese hamster ovary (CHO) cells were cultured in Ham's F12 nutrient mixture (Wako) supplemented with $10 \%$ fetal bovine serum (FBS) at $37{ }^{\circ} \mathrm{C}$ and $5 \% \mathrm{CO}_{2}$. The mouse ES cells were maintained on feeder cells in an ES cell medium consisting of Dulbeccos' modified Eagle's medium (DMEM) (Kohjin Bio) supplemented with $20 \%$ FBS, $0.1 \mathrm{mM}$ nonessential amino acids (Gibco), $2 \mathrm{mM}$ glutamine (Gibco), $1000 \mathrm{U} / \mathrm{ml}$ ESGRO (Chemicon), and $0.1 \mathrm{mM} \beta$ mercaptoethanol (Sigma).

\section{Microcell-mediated chromosome transfer}

Microcell-mediated chromosome transfer (MMCT) from $\mathrm{CHO}$ cells to mouse ES cells was carried out as described previously (Suzuki et al. 2006). Briefly, twenty 10-cm dishes of $\mathrm{CHO}$ cells were grown to $70 \%$ confluency and Colcemid (Wako) added to $0.05 \mu \mathrm{g} / \mathrm{ml}$. The cells cultured for $72 \mathrm{~h}$ were harvested by trypsinization and resuspended in pre-warmed serum-free DMEM (Wako) containing Cytochalasin B (Calbiochem) at a final concentration of $20 \mu \mathrm{g} / \mathrm{ml}$. The suspension was incubated, and then an equal volume of Percoll (Amersham Biosciences) was added. The suspension was centrifuged in a Hitachi R20A2 rotor at $15,000 \mathrm{rpm}$ for $90 \mathrm{~min}$ at $37{ }^{\circ} \mathrm{C}$. The microcell fraction containing the HLA-HAC was mixed with ES cells. After centrifugation at $2000 \mathrm{rpm}$ for $5 \mathrm{~min}$, the pellet was suspended in $1 \mathrm{ml} 50 \%$ PEG1500 (Roche). The fusion product was washed and plated onto three $10-\mathrm{cm}$ dishes layered with feeder cells. ES cells containing the HLA-HAC were selected with $150 \mu \mathrm{g} / \mathrm{ml}$ G418 (Sigma).

Generation of chimeric mice

Chimeric mice were produced from mouse ES cell lines. The ES cells were aggregated with eight-cell embryos derived from BDF2 mice and then transferred into pseudopregnant ICR females. Almost $100 \%$ coat color chimeric mice were mated with C57BL/6 (B6) mice (Jackson Laboratory) to obtain transgenic mice. All animal experiments were approved by the Institutional Animal Care and Use Committee of RIKEN RCAI and Kazusa DNA Research Institute.

\section{HLA-DR gene constructs}

We used a whole CTD-2052L14 BAC clone $(100 \mathrm{~kb})$ as the HLA-DRA gene because this BAC contains only the HLADRA gene (referenced on NCBI CloneDB browser; http:// www.ncbi.nlm.nih.gov/clone/79827/). The DRA-BAC used in this study contains an upstream $22-\mathrm{kb}$ region and the HLA-DRA gene (Fig. 1a). Only a 268-base pair region of the 5 '-flanking region of the HLA-DRA gene has been reported to be sufficient for the cell type-specific expression on the transgenic mouse as a multi-copy in this case (Fukui et al. 1993). So, we included the longer upstream region. On the other hand, for the cis-elements of the DRB1 gene, consensus has not yet been reached; therefore, we used the maximum length of the upstream region, close to the border of the next pseudo gene. A HLA-DRB $1 * 0405$ BAC was constructed from a $28.5-\mathrm{kb}$ upstream region of the HLA-DRB $1 * 0103$ gene in a RP11-379F19 BAC clone (referenced on NCBI CloneDB browser; http://www.ncbi.nlm.nih.gov/clone/ 312446/) and genomic DNA (IHW09415) containing all exons of HLA-DRB $1 * 0405$ by using a Red recombination with pBADTcTypeG plasmids whose mutant $\operatorname{Red} \alpha$ and mutant Red $\beta$ proteins increase the recombination efficiency (Nakayama and Ohara 2005). Consequently, the 50-kb BAC had $28.5 \mathrm{~kb}$ upstream of the translational start site and all exons and introns of the HLA-DRB1*0405 gene (Fig. 1a). The HLA-DRA and DRB1 BACs were modified by addition of the lox66/puromycin resistance cassette or lox66/ blasticidin $\mathrm{S}$ resistance cassette, respectively (Fig. 1), in place 


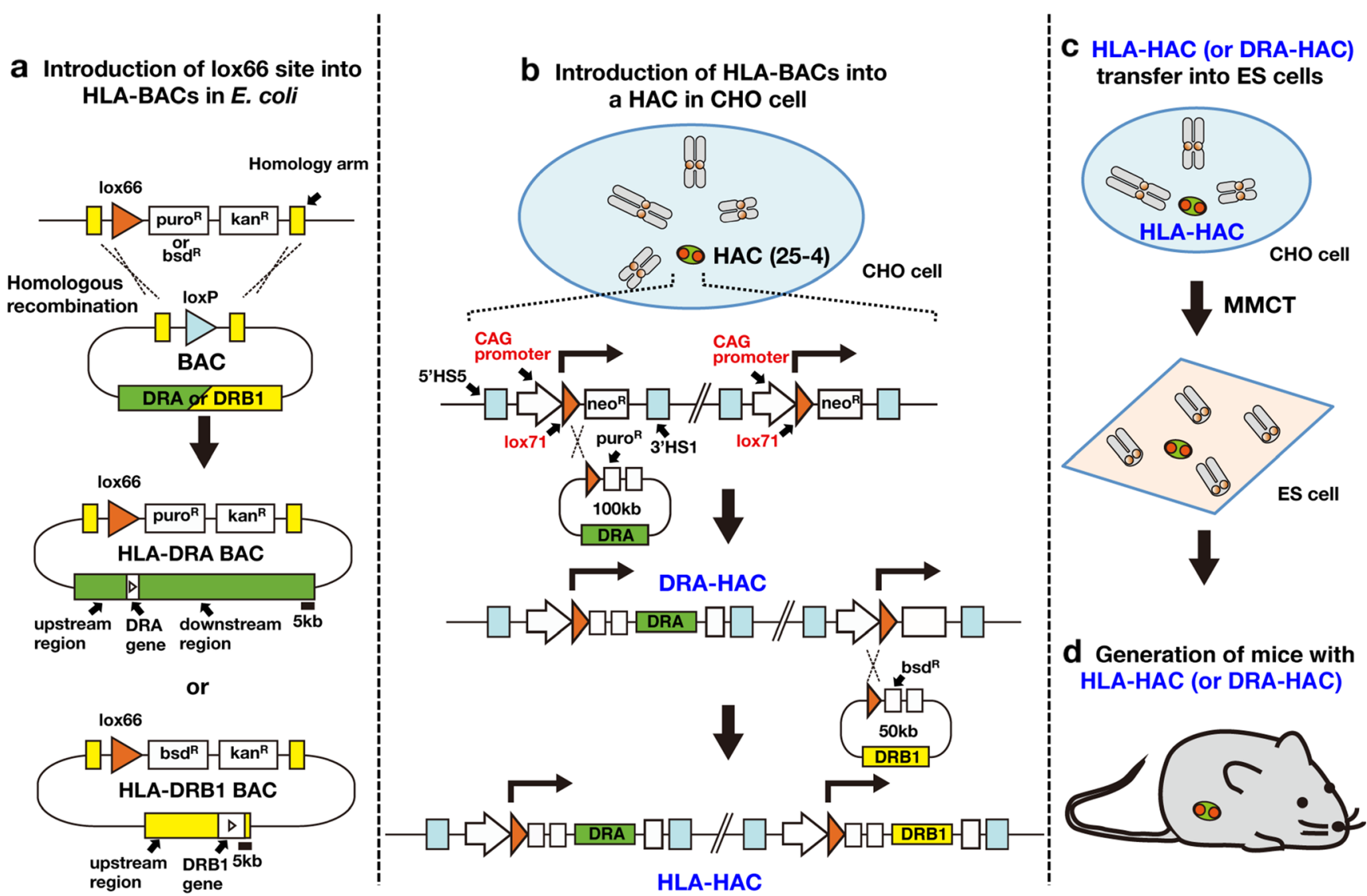

Fig. 1 Generation of mice with HACs containing human genes. a Introduction of the lox66 site into the HLA-BAC by Red recombination in $E$. coli. $\mathbf{b}$ Schematic representation of two consecutive introductions of HLA-BACs into a HAC vector using Cre/lox recombination. The gene in the entry vector was inserted into a $\mathrm{HAC}$ vector in $\mathrm{CHO}$ cells by $\mathrm{Cre} / \mathrm{lox}$

of loxP by the Red recombination in Escherichia coli according to a previously described method (Ikeno et al. 2009).

\section{DNA transfection}

For the insertion of the HLA-DR BACs into the HAC vector, $1 \mu \mathrm{g}$ of the HLA-DR BAC DNA was co-transfected with $0.5 \mu \mathrm{g}$ of CAGGS-Cre into $\mathrm{CHO}$ cells $\left(5 \times 10^{5}\right)$ retaining the HAC vector (Ikeno et al. 2009) with FuGENE HD (Promega) according to the manufacturer's instructions. DRA cell lines were selected with $6 \mu \mathrm{g} / \mathrm{ml}$ puromycin (Sigma) and DRB1 cell lines with $3 \mu \mathrm{g} / \mathrm{ml}$ blasticidin S (Wako).

Real-time quantitative PCR analysis

Total RNA was isolated using the RNeasy Micro Kit (Qiagen). cDNA was synthesized using the Verso cDNA Synthesis Kit (Thermo Scientific), and 25-ng aliquots were used for PCR. Real-time PCR was carried out using the ABI 7500 Real-Time PCR System (Applied Biosystems). PCR reactions were carried out using the Luminaris Probe Low ROX qPCR Master recombination. Successful recombinants were selected by puromycin (puro) or blasticidin (bsd) resistance. c HLA-HAC transfer from $\mathrm{CHO}$ cells to mouse ES cells was achieved by MMCT. $\mathbf{d}$ Chimeric mice with HLA-HAC were created by aggregation of the ES cells with BDF2 eightcell embryos

Mix (Thermo Scientific) and each assay mix: Hs00219578_m1 (HLA-DRA), Hs00830030_sH (HLADRB1), or Mm99999915 g1 (mouse GAPDH). The PCR protocol was $95{ }^{\circ} \mathrm{C}$ for $10 \mathrm{~min}$ and 40 cycles of $95^{\circ} \mathrm{C}$ for $15 \mathrm{~s}$ and $60^{\circ} \mathrm{C}$ for $60 \mathrm{~s}$.

Genomic PCR analysis

Genomic DNA was extracted from mouse tails using a Wizard Genomic DNA Purification Kit (Promega) and PCR performed as follows. The amplification conditions were $98{ }^{\circ} \mathrm{C}$ for $1 \mathrm{~min}$, followed by 35 cycles of $98^{\circ} \mathrm{C}$ for $10 \mathrm{~s}, 60^{\circ} \mathrm{C}$ for $30 \mathrm{~s}$, and $72{ }^{\circ} \mathrm{C}$ for $30 \mathrm{~s}$. The following primers were used: HLA-DRA-1, 5'-CACGAACAGCCCTGTGGAAC-3' and 5'-CTCAGTTGAGGGCAGGAAGG-3'; HLA-DRA-2, 5'TCTCCCAGAGACTACAGAGAACG- $3^{\prime}$ and $5^{\prime}$-CCTGCG TTCTGCTGCATTG-3'; HLA-DRB1-1, 5'-TCATTTCTTC AACGGGACGGAG- ${ }^{\prime}$ and $5^{\prime}$-TGCACTGTGAAGCTCT CACC-3'; HLA-DRB1-2, 5'-TGGTCTGCTCTGTGAA TGG- ${ }^{\prime}$ and $5^{\prime}$-TCCACTGTGAGAGGGCTCATC-3'; HLADRB1-3, 5'-GTGGGAGATGCAGACTTGTGG-3' and 5'- 
TGCTTCTTCCTCATCATCTCTGC-3'; and HLA-DRB1-4, 5'-GAACCAACACACCGACGGATAG-3' and 5'-GAGG GATTGGACACAGAGATCAG-3'.

\section{Fluorescent in situ hybridization}

Fluorescent in situ hybridization (FISH) analysis was carried out according to conventional procedures. To detect HACs, biotin-labeled $\alpha 21-\mathrm{I}$ alphoid DNA (11-4) (Ikeno et al. 1994) and digoxigenin-labeled pBelo-BAC were used as probes. For dual FISH, biotin-labeled DNA was visualized with FITCconjugated avidin (Vector) and digoxigenin-labeled DNA with TRITC-conjugated antidigoxigenin antibody (Roche).

\section{Preparation of spleen cells}

Splenocytes were smashed with two slide glasses, filtered through a 70- $\mu \mathrm{m}$ cell strainer, and washed with Roswell Park Memorial Institute (RPMI) medium (Wako). The supernatant was removed and the red blood cells were destroyed with Red Blood Cell Lysing Buffer Hybri-Max (Sigma). Cells were washed with RPMI medium containing $10 \% \mathrm{FBS}$ and then resuspended in the same medium.

\section{Flow cytometry}

Cells were stained with the antibody against HLA-DR conjugated with Alexa Fluor 488 (50 $\mu \mathrm{g} / \mathrm{ml}$, L243, BioLegend) for $30 \mathrm{~min}$ on ice. Flow cytometric analysis was performed using a FACS Calibur instrument (BD Biosciences) and the results analyzed using the FlowJo software program (Tree Star).

\section{Results}

Introduction of HLA-BACs into a HAC vector in $\mathrm{CHO}$ cells

The HLA-BACs with lox66/puromycin or blasticidin S resistance cassette were inserted into a $\mathrm{HAC}$ vector in $\mathrm{CHO}$ cells by $\mathrm{Cre} / \mathrm{lox}$ recombination. Successful Cre-lox recombination between the lox66 site at the promoterless cassette (puro or bsd) on the BAC and the lox71 site of the gene expression cassette on the 25-4 HAC vector produced drug-resistant cell lines (puro or bsd; Fig. 1). PCR analysis of drug-resistant cells after the first insertion of the HLA-DRA BAC into the 25-4 HAC showed that 22 of 31 cell lines had successful insertion. The second insertion of the HLA-DRB $1 * 0405$ BAC was performed using the first insertion-positive cell line (No. 11; Fig. 2a). The 25-4 HAC vector carrying HLA-DRA BAC (DRA-HAC) and carrying both HLA-DRA BAC and HLADRB1*0405 BAC (HLA-HAC) was stably maintained as an extra chromosome through Cre-lox reactions in $\mathrm{CHO}$ cells (Fig. 2b).

Transfer of the HACs into mouse ES cells by MMCT

We used mouse ES cells $\left(1 \times 10^{7}\right.$ cells $)$ and microcells prepared from $\mathrm{CHO}$ cells $\left(1 \times 10^{7}\right.$ cells $)$ containing DRA-HAC or HLA-HAC for the MMCT (Doherty and Fisher 2003, Fig. 1c). We obtained over 70 mouse ES cell colonies screened by G418 resistance and analyzed 10 colonies by FISH. The majority of each ES cell lines carried a single copy of the HAC with no integration signal of the HAC DNA in the mouse chromosomes (Fig. 3a, b). Karyotype analysis showed that the number of cells with a normal mouse karyotype (retaining 40 mouse chromosomes) varied between 30 and $100 \%$ among ES cell lines (Fig. 3b). We used cell line No. 9 because 10 of 10 analyzed cells showed normal karyotypes and contained a single HLA-HAC for chimeric mouse production.

Chimeric mice carrying the HACs

Chimeric mice carrying the DRA-HAC or HLA-HAC were created by aggregation of the ES cells with BDF2 eight-cell embryos. Three male mice with a high degree of chimerism (almost $100 \%$ in coat color, Fig. 3c) were investigated for germ line transmission of the HLA-HAC. After crossing the chimeric male mice with five female B6 mice, 6-week-old F1 offspring (39 F1 mice) were analyzed by PCR using HACspecific primers; 35.3-58.3\% of these F1 offspring were positive for the HAC signals (Table 1). Thus, HLA-HACs were transmitted through the germ line of all of the chimeric mice we examined, and a total of $46.2 \%$ of F1 offspring (18 of 39 ) possessed the HLA-HAC (maximum expected value was $50 \%$ ). On the other hand, three chimeric male mice harboring the DRA-HAC were crossed with six female B6 mice; 47.1$53.3 \%$ of these F1 offspring were positive for the HAC signals (Table 1). A total of $49.1 \%$ of F1 offspring (26 of 53) possessed the DRA-HAC. Because the ES cell line used in this experiment was selected carefully by karyotype analysis and HAC retention rate, we effectively obtained chimeric mice with chimeric rates and HAC transmission rates that were high enough.

Mitotic stability of the HACs in mouse somatic tissues

To investigate the mitotic stability of HACs in the somatic tissues of mice, the retention of HLA-HAC in cells from the bone marrow, brain, heart, kidney, liver, spleen, and tail of 7 months old F1 mice was analyzed by FISH. As expected, the HLA-HACs were retained in all of these tissues (Fig. 4a, b). The retention rate in the brain, heart, kidney, liver, and tail at 7 months (highest individual (male) 90.0-96.7\%, lowest 
Fig. 2 HLA-BAC introduction into a HAC vector in $\mathrm{CHO}$ cells. a PCR analysis of two consecutive introductions of HLA-BACs into a $\mathrm{HAC}$ vector using site-specific recombination. The first and second introductions of BAC were confirmed by PCR with the CAG promoter and puro sequences (22 positive in 31 analyzed) and the CAG promoter and bsd sequences (19 positive in 20 analyzed), respectively. b FISH analysis of the HLA-HAC (arrowhead) in CHO cells. This HAC carried a single copy of HLA-DRA BAC and a single copy of HLA-DRB1 BAC, confirmed by a further insertion of a DNA fragment to the remaining cassettes on the HAC and PCR analysis (data not shown). The red signal shows BAC DNA, the green signal shows alphoid DNA and the white signal shows DNA counterstained with DAPI

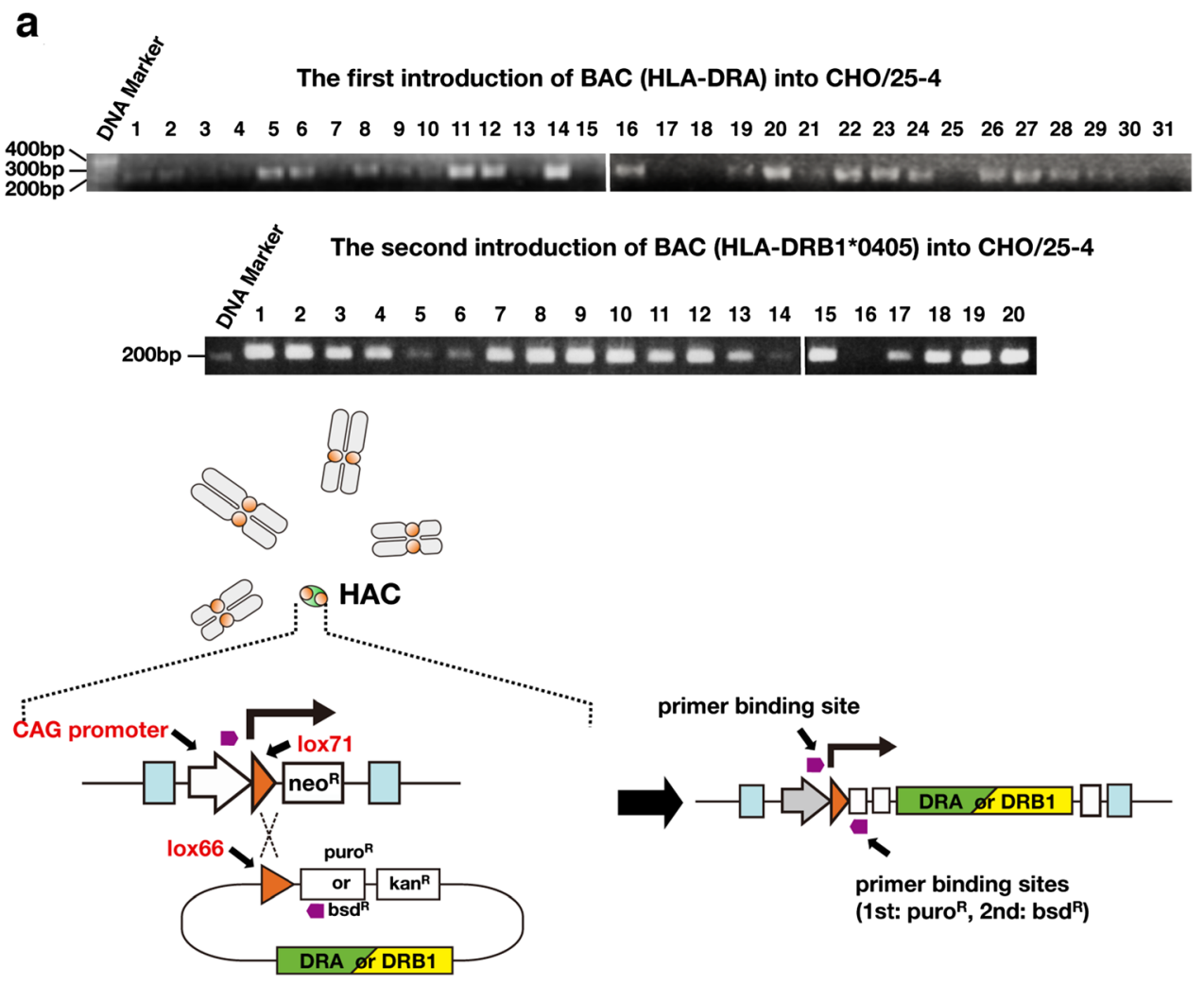

b
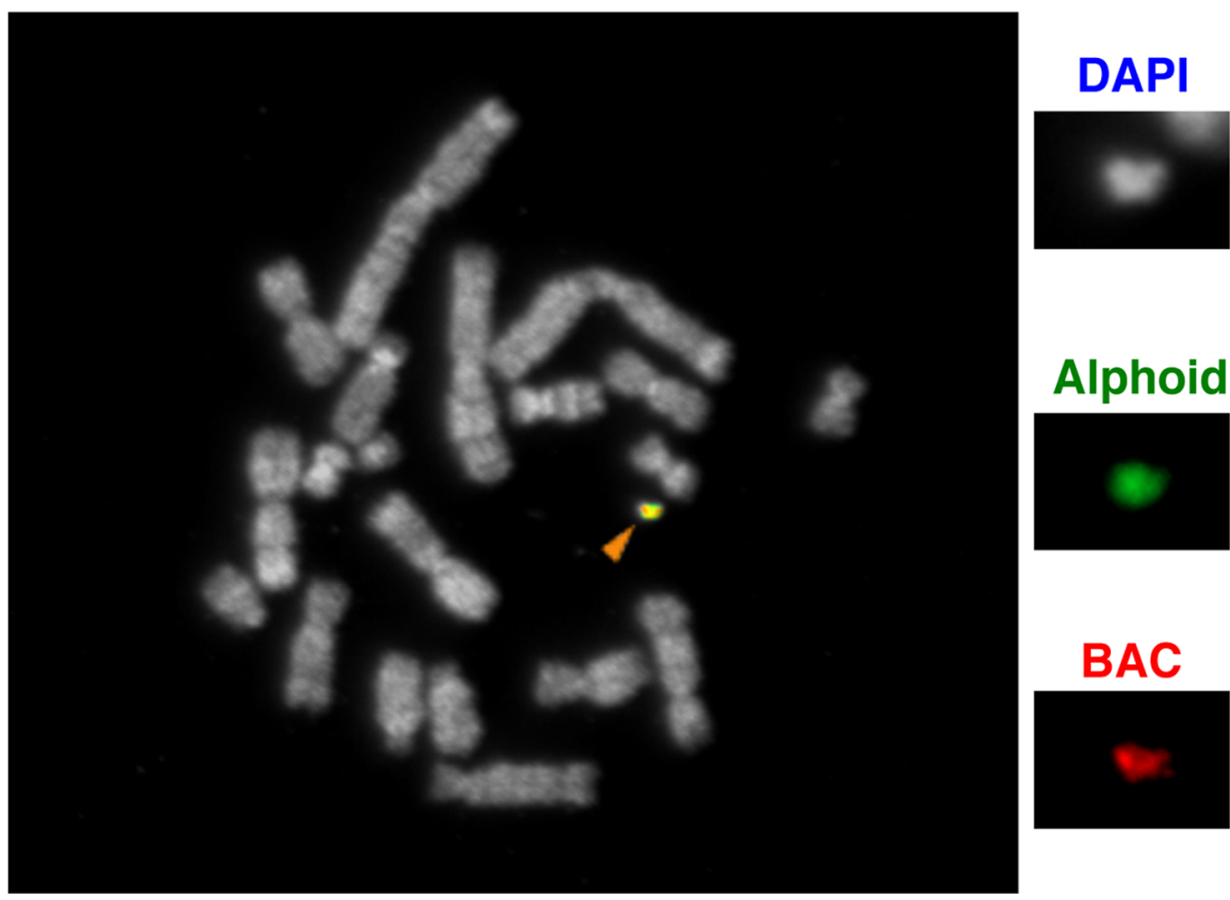

Alphoid

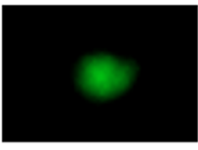

\section{BAC}

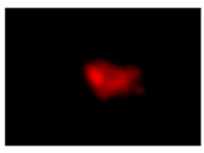

individual (female) 56.7-73.3\%) was similar to that of tail cells from a 6-week-old mouse (highest individual $96.7 \%$, lowest individual $60.0 \%$; Table 2). In contrast, the HAC retention rate of the bone marrow and spleen was lower than that of the other tissues (highest individual 73.3 and $76.7 \%$, lowest individual 43.3 and $40.0 \%$, respectively). The HAC 
Fig. 3 Generation of TMC mice. a FISH analysis of the HLA-HAC in mouse ES cells. The mouse ES cell lines carrying HLA-HAC (arrowhead) were established by MMCT. The red signal shows BAC DNA, the green signal shows alphoid DNA and the white signal shows DNA counterstained with DAPI. b Karyotype analysis of mouse ES cells harboring HLA-HAC. An asterisk $(*)$ indicates the clone used for generation of the TMC mouse. c Chimeric mouse from mouse ES/HLA-HAC. The coat color of the mouse ES cell is agouti. This mouse is almost $100 \%$ ES cell-derived

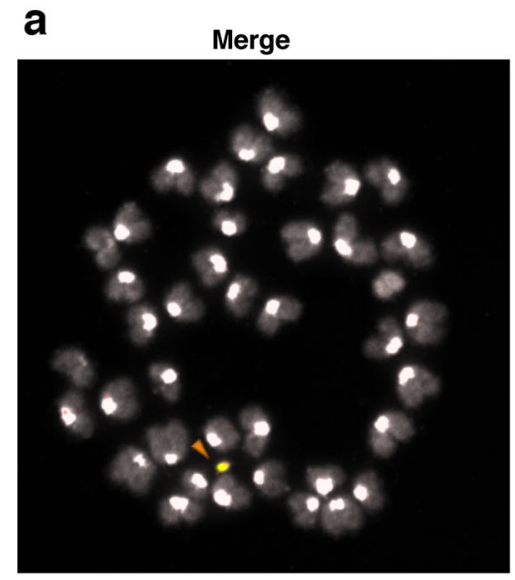

b

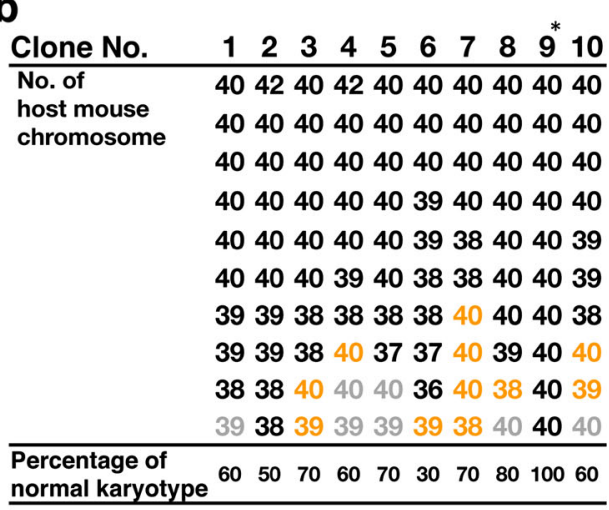

1HAC/cell, 2HACs/cell, no HAC/cell

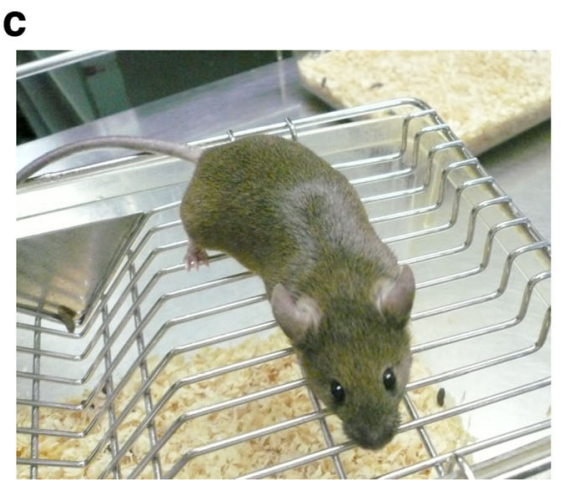

retention rates in the tissues varied among F1 mice, but they were represented in tail cells.

Retention and transmission of the HACs through mouse generations

To investigate the retention and transmission rates of HACs in the TMC mouse and the stability of the large insert genes on the HAC through mouse generations, F1 mice were

Table 1 HAC transmission rate for six chimeric mice

\begin{tabular}{llll}
\hline Parental origin & $\begin{array}{l}\text { Total } \\
\text { offspring }\end{array}$ & $\begin{array}{l}\text { Total } \\
\text { HAC + mice }\end{array}$ & $\begin{array}{l}\text { HAC }+ \\
\text { mice/offspring } \\
(\%)\end{array}$ \\
\hline HLA-HAC male No. 1 & 12 & 7 & 58.3 \\
HLA-HAC male No. 2 & 10 & 5 & 50.0 \\
HLA-HAC male No. 3 & 17 & 6 & 35.3 \\
HLA-HAC male total & 39 & 18 & 46.2 \\
DRA-HAC male No. 4 & 15 & 8 & 53.3 \\
DRA-HAC male No. 5 & 17 & 8 & 47.1 \\
DRA-HAC male No. 6 & 21 & 10 & 47.6 \\
DRA-HAC male total & 53 & 26 & 49.1 \\
\hline
\end{tabular}

backcrossed with B6 mice for seven more generations for a total of eight backcrosses. "The HAC transmission" of all pups was examined by PCR analysis of genomic DNA extracted from the 3-mm tail of each mouse with HAC-specific primers. "The retention rate of the HAC" in tail cells from the PCR positive mouse was examined by FISH analysis. (The PCR band-negative mice did not show any HAC FISH signal.) In F1 mice, the retention rates of HLA-HAC and DRAHAC were similar on average but variable in individual mice (average 82.6 and $86.2 \%$, respectively; Tables 2 and 3). The retention rate of HLA-HAC in tail cells through mouse generations was $70-80 \%$, whereas the retention rate of DRAHAC was around $90 \%$ (Fig. 5a). Unexpectedly, a significant difference was observed in the transmission rate between the two types of HACs through mouse generations. The transmission rate of DRA-HAC was very close to the maximum expected value of $50 \%$ (average $45.9 \%, 61 / 133$ mice) through eight generations, but the transmission rate of HLA-HAC varied in lower ranges (average $23.5 \%$, 38/ 162 mice, Fig. 5b). Clearly, the additional insertion of the DRB1 BAC into another gene expression cassette site on the HLA-HAC caused some instability in the HAC through the mouse generations. However, all HACs were detected as extra-chromosomal signals independently from endogenous mouse chromosomes, though we observed 
Fig. 4 Characteristics of transgenic mice harboring HLAHAC. a HLA-HAC retention rate in the bone marrow, brain, heart, kidney, liver, spleen, and tail of a 7-month-old F1 mouse. The percentage of HAC-retaining cells was calculated from 60 cells. b Metaphase chromosomes from the spleen and interphase nuclei from the heart of a 7-month-old F1 mouse (the highest HAC retention male in Table 2) were analyzed by probing for alphoid and BAC DNA in FISH a

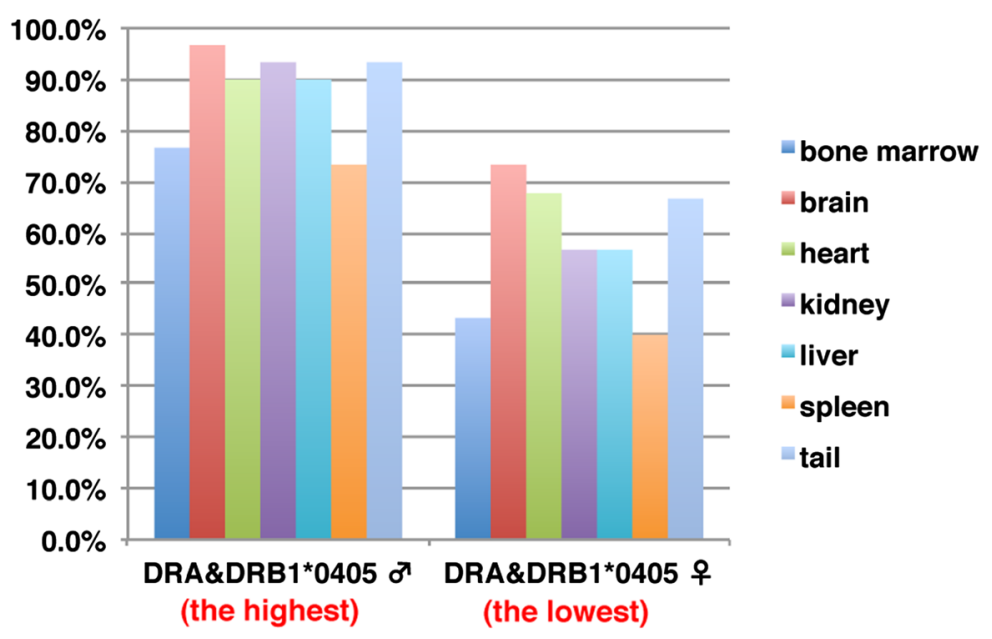

b
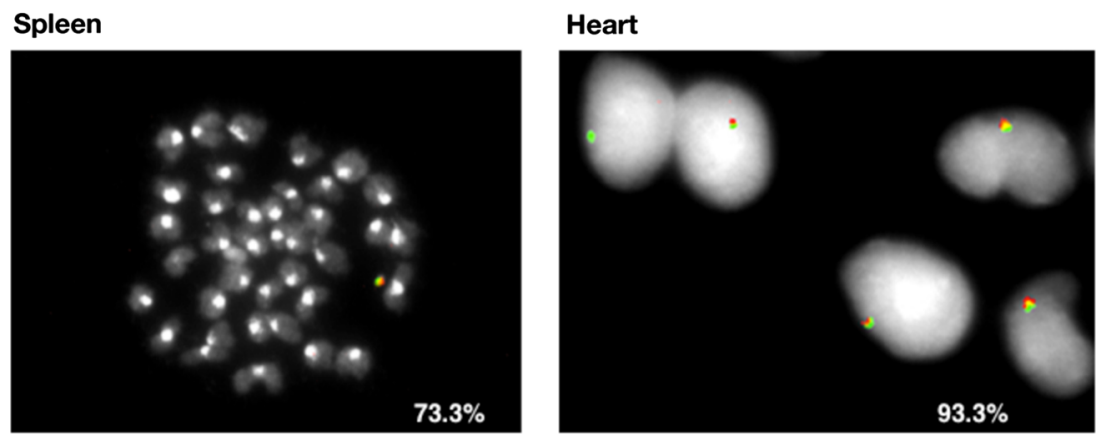

Alphoid BAC

over 6000 cell samples in the FISH analyses. We also confirmed by genomic PCR that the structures of the HLA genes on the HACs were stably maintained through HAC transfer into the mouse and eight consecutive mouse generations (Supplemental Fig. S1).

Functional expression of HLA-DR genes from the HLA-HAC

The expression of HLA-DRA and HLA-DRB1 as cell surface antigens was evaluated by flow cytometry using splenocytes from chimeric mice and anti-HLA-DR antibody. The cell surface signals were detected specifically from the chimeric mouse carrying the HLA-HAC (13.5\% of cell population, Fig. 6a). Because the antibody binds a conformational epitope on HLA-DR $\alpha$ that depends on the correct folding of the $\alpha \beta$ heterodimer (Moro et al. 2005), the result clearly indicates that the HLA-DRA and HLA-DRB1*0405 genes from the HLA$\mathrm{HAC}$ were expressed and folded as a $\alpha \beta$ heterodimer on the cell surface of splenocytes in the chimeric mouse. Functional expression was also observed in the eighth-generation TMC mice ( $18.9 \%$ of cell population, Fig. $6 \mathrm{~b}$ ), indicating that such functional expression of the human genes on the HAC was maintained through mouse generations. It was reported that only about a half population $(51.6 \%)$ of splenocytes was expressing endogenous class II MHC on their cell surface by flow cytometric analysis in B6 wild-type mice (Jux et al. 2013). According to this data, an expected population of the splenocytes expressing the HLA from the HAC in the chimeric mouse is estimated to be approximately 20-37\% (40$73 \%$ splenocytes retain the HLA-HAC; Fig. 4a) and that in F8 mouse is approximately $22-30 \%(44-60 \%$ splenocytes retain the HLA-HAC estimating from the $74 \%$ HAC retention rate in the tail cells; Fig. 5a). The actual measurements of the cell surface signals by flow cytometry using splenocytes are $13.5 \%$ in the chimeric mouse and $18.9 \%$ in the F8 mouse. These values, more than a half or very close to the expected values estimated from mouse endogenous class II MHC expression, are significant enough for considering transgenic human class II MHC genes on the HAC vector in mice through generations. 
Table 2 HAC retention rate in the tail cells of 6 weeks old F1 HLA-HAC mice

\begin{tabular}{|c|c|c|c|}
\hline & $\mathrm{HAC}$ retention rate $(\%)$ & Sex of offspring & Parental origin \\
\hline & 60.0 & $q^{\mathrm{b}}$ & No. $2{ }^{\lambda}$ \\
\hline & 66.7 & $\hat{\sigma}$ & No. $1 \overbrace{}^{\pi}$ \\
\hline & 66.7 & 우 & No. $2 \widehat{\diamond}$ \\
\hline & 73.3 & $\hat{\sigma}$ & No. $3{ }^{\pi}$ \\
\hline & 76.7 & 우 & No. $3{ }^{\lambda}$ \\
\hline & 76.7 & $\hat{\sigma}$ & No. $1 \overbrace{}^{\pi}$ \\
\hline & 80.0 & $\delta^{1 \mathbf{a}}$ & No. $2{ }^{\lambda}$ \\
\hline & 83.3 & $\hat{\sigma}$ & No. $3{ }^{\lambda}$ \\
\hline & 86.7 & 우 & No. $3 \widehat{\jmath}$ \\
\hline & 86.7 & 우 & No. $3{ }^{\lambda}$ \\
\hline & 86.7 & $\hat{\sigma}$ & No. $3{ }^{\lambda}$ \\
\hline & 86.7 & $\hat{\sigma}$ & No. $1 \overbrace{}^{\wedge}$ \\
\hline & 90.0 & q & No. $1 \sigma^{\pi}$ \\
\hline & 90.0 & 우 & No. $2 \widehat{\jmath}$ \\
\hline & 90.0 & $\hat{\sigma}$ & No. $2 \widehat{\jmath}$ \\
\hline & 93.3 & 우 & No. $1{ }^{\lambda}$ \\
\hline & 96.7 & $\partial^{b}$ & No. $1{ }^{\lambda}$ \\
\hline & 96.7 & $\hat{\sigma}$ & 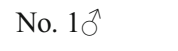 \\
\hline Average & 82.6 & & \\
\hline
\end{tabular}

${ }^{a}$ The first mouse used for HAC transmission through eight generations

${ }^{\mathrm{b}}$ Mice used for analyzing the HAC retention rate in the bone marrow, brain, heart, kidney, liver, spleen, and tail of 7-month-old F1 mice (see also Fig. 4a, b)

Tissue-specific expression of HLA-DR genes from the HAC in mice

The expression of MHC class II molecules is basically confined to immunocompetent cells, such as B cells, macrophages, dendritic cells, and thymic epithelial cells. To determine whether the HLA-DR genes on the HAC were expressed in a tissue-specific manner in eighth-generation TMC mice, total cellular RNA was extracted from various tissues and examined by real-time quantitative PCR analysis. The results show that the expression pattern was similar between the two individuals (Fig. 7 and Supplemental Fig. S2). The expression levels of the HLA-DRA and HLA-DRB1 genes were high in the lung, spleen, lymph node, and thymus, but they were extremely low in the liver, kidney, and bone marrow. These results indicate that the natural patterns of tissue-specific expression of the human HLA-DRA and HLA-DRB $1 * 0405$ genes were almost mimicked on the HAC through the mouse generations. On the other hand, the expression pattern of these two genes on the HAC in CHO and ES cells was stochastic (Supplemental Fig. S3). The No. 9 ES cell line used for chimeric mouse production showed little, if any, HLA-DRA and HLA-DRB $1 * 0405$ expression. Thus, human HLA-DR genes including regulatory elements and promoters used in
Table 3 HAC retention rate in tail cells from 6-week-old F1 DRA-HAC mice

\begin{tabular}{|c|c|c|c|}
\hline & HAC retention rate $(\%)$ & Sex of offspring & Parental origin \\
\hline & 63.3 & $\hat{\jmath}$ & No. $4{ }^{\curvearrowright}$ \\
\hline & 66.7 & $\hat{\jmath}$ & No. $60^{\lambda}$ \\
\hline & 76.7 & q & No. $4 \lesssim$ \\
\hline & 80.0 & q & No. $5 \hat{\jmath}$ \\
\hline & 80.0 & $q$ & No. $60^{\lambda}$ \\
\hline & 83.3 & q & No. $4{ }^{\lambda}$ \\
\hline & 83.3 & $\hat{0}$ & No. $4{ }^{\lambda}$ \\
\hline & 83.3 & $\hat{0}$ & No. $50^{\lambda}$ \\
\hline & 83.3 & $q$ & No. $60^{\lambda}$ \\
\hline & 83.3 & $\hat{\sigma}$ & No. $60^{\lambda}$ \\
\hline & 86.7 & $\hat{\sigma}$ & No. $4 \hat{\circ}$ \\
\hline & 86.7 & $\hat{0}$ & No. $5 \hat{\jmath}$ \\
\hline & 90.0 & q & No. $4 \widehat{\jmath}$ \\
\hline & 90.0 & $q$ & No. $5 \hat{\circ}$ \\
\hline & 90.0 & $q$ & No. $5 \hat{\jmath}$ \\
\hline & 90.0 & $\hat{\sigma}$ & No. $5{ }^{\AA}$ \\
\hline & 90.0 & $\hat{0}$ & No. $5 \hat{\jmath}$ \\
\hline & 90.0 & q & No. $6{ }^{\lambda}$ \\
\hline & 90.0 & q & No. $6 \widehat{0}$ \\
\hline & 90.0 & $\hat{0}$ & No. $6{ }^{\lambda}$ \\
\hline & 93.3 & q & No. $4{ }^{\lambda}$ \\
\hline & 93.3 & $\hat{\jmath}$ & No. $4{ }^{\Uparrow}$ \\
\hline & 93.3 & q & No. $6{ }^{\lambda}$ \\
\hline & 93.3 & q & No. $60^{\lambda}$ \\
\hline & 93.3 & $\delta^{a}$ & No. $6 \widehat{0}$ \\
\hline & 96.7 & $\hat{0}$ & No. $5 \AA$ \\
\hline Average & 86.2 & & \\
\hline
\end{tabular}

${ }^{\mathrm{a}}$ The first mouse used for HAC transmission through eight generations

this study acquired precise expression epigenetically in specific cell types through the mouse developmental process.

\section{Discussion}

We generated transgenic mice using a HAC carrying two single-copy HLA-DR genes. For the past 20 years, many HLA-DR transgenic mice, including those with an immunodeficient NOG background, have allowed the analysis of HLA-restricted responses with variable success (Fukui et al. 1993; Suzuki et al. 2012). This study is the first report of transgenic mice carrying two single-copy human genes composed of human regulatory elements and exon-intron structures. Both the HLA-DRA and HLA-DRB1 genes on the HAC were expressed in a tissue-specific manner and exhibited functional expression as a cell surface receptor in the TMC mouse. The tissue-specific expression pattern of HLA-DR in 
a

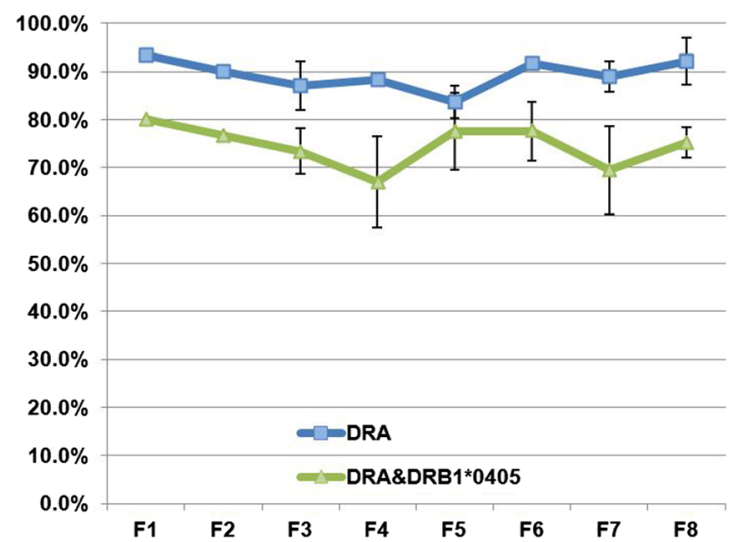

Fig. 5 The retention and transmission rates of HAC. a The retention rate of HAC in tail cells was determined by FISH analysis using alphoid and BAC DNA probes. The percentage of HAC-retaining cells was calculated

the TMC mouse was similar to that of endogenous class II MHC genes in mice. The mRNA expression level of the class II MHC in mice was high in the spleen and thymus, but it was barely detectable in the liver, kidney, and bone marrow (Pinkert et al. 1985). A previous report of a mouse carrying 12 copies of $24 \mathrm{~kb}$ of the HLA-DRA gene showed that the transgene was overexpressed in some tissues and moderately in other tissues (Altmann et al. 1993). In another transgenic mouse, a 6-kb HLA-DRA gene fragment was integrated into the $\mathrm{X}$ chromosome and subjected to random inactivation (Fukui et al. 1993). Based on these observations, our present analysis indicates that the features of $\mathrm{HAC}$, an episomal vector with a large DNA capacity and a controllable gene number, were exploited effectively for the production of HLA-DR transgenic mice. Furthermore, HLA-HACs were transmitted through at least eight generations, and HLA-DR genes on the HAC maintained functional expression during that time. We also confirmed that two human genes that were reorganized on the HAC were cooperatively expressed in immunocompetent

\section{Chimeric mouse}

B6 (control)

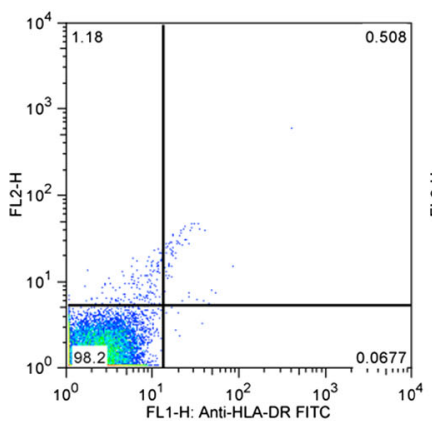

HLA-HAC

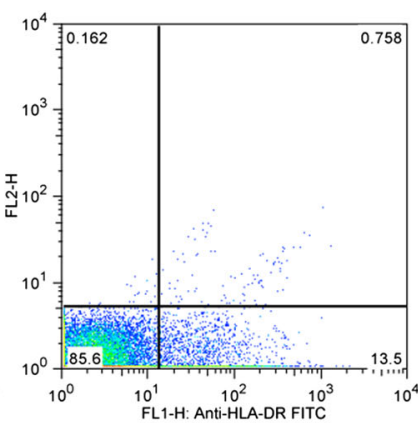

Fig. 6 Flow cytometric analysis of surface molecules on splenocytes from a chimeric mouse and F8 generation mouse. Cells were stained with anti-HLA-DR, L243. A B6 mouse with no HAC was used as a negative b

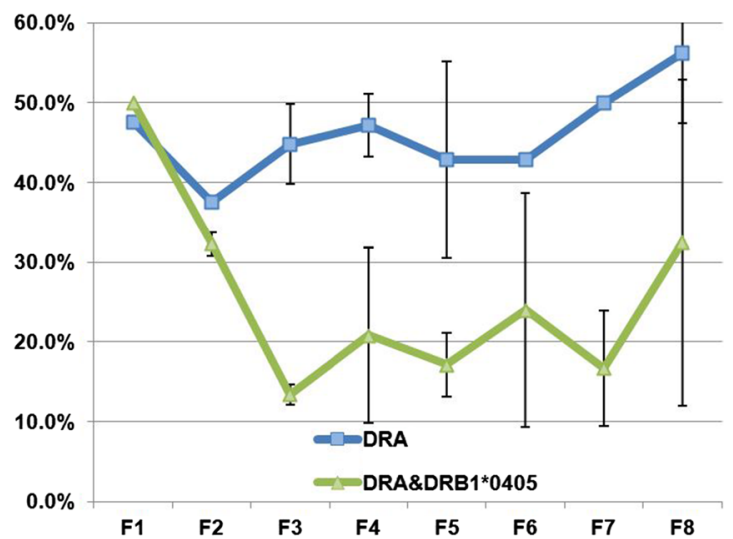

from 60 cells. Error bars indicate standard deviations. b An average of each transmission rate was determined by more than two crossings

cells in mice. Taking account of these results, although we checked only small fragments of the genes on the HAC by PCR (Supplemental Fig. S1), it is conceivable that at least no major rearrangement influencing the functional expression was observed on the HACs through eight mouse generations.

There are over 40 haplotypes of HLA-DRB, but only one functional type of HLA-DRA in the Japanese population. We have already generated a DRA-HAC mouse that harbors a HAC vector carrying only an HLA-DRA locus. Recently, the novel transgenic technique pronuclear injection-based targeted transgenesis (PITT) was reported (Ohtsuka et al. 2010; Ohtsuka et al. 2012). The PITT technique enables targeted integration of a single-copy transgene into a predetermined locus by Cre-loxP-mediated recombination and guarantees a stable and reproducible transgene expression. A combination of the DRA-HAC mouse and such a technique will allow the generation of more HLA-DR haplotypes.

\section{The 8th filial generation mouse}

\section{5-4 HAC (B6) (control)}

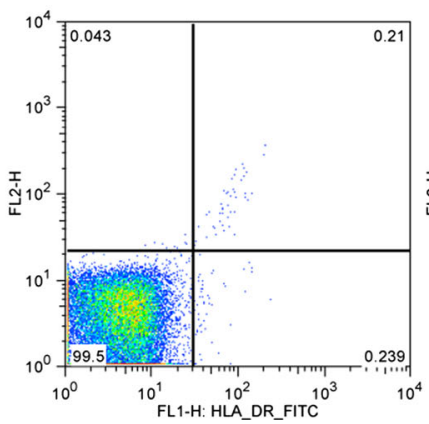

HLA-HAC

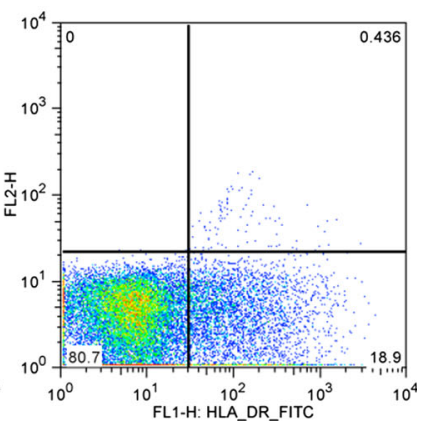

control for the chimeric mouse, and the B6 background for five filial generations with HAC (no inserts) was used as a negative control for the F8 generation mouse 


\section{The 8th filial generation HLA-HAC mouse No.1}

human HLA-DRA/mouse Gapdh

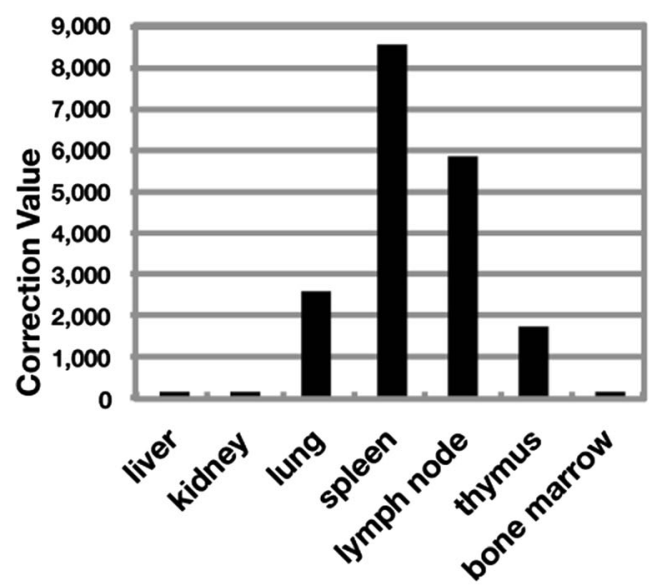

human HLA-DRB1*0405/mouse Gapdh

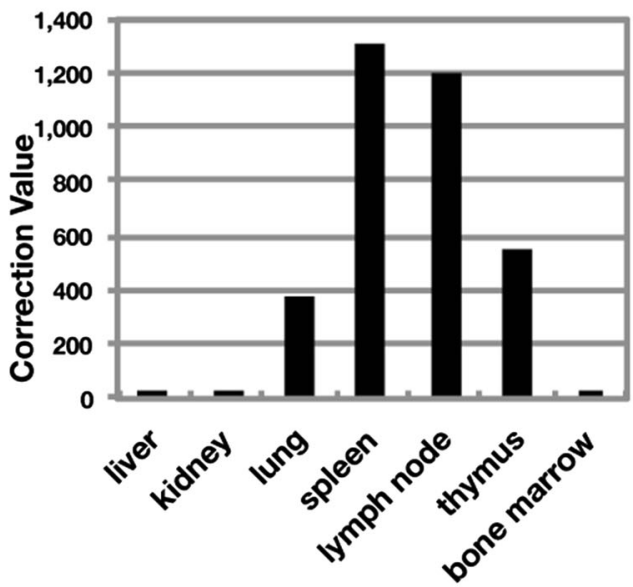

The 8th filial Generation HLA-HAC mouse No.2

human HLA-DRA/mouse Gapdh

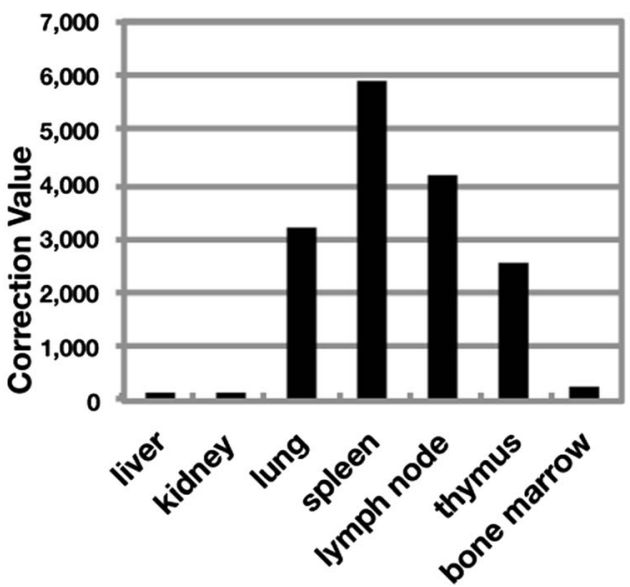

human HLA-DRB1*0405/mouse Gapdh

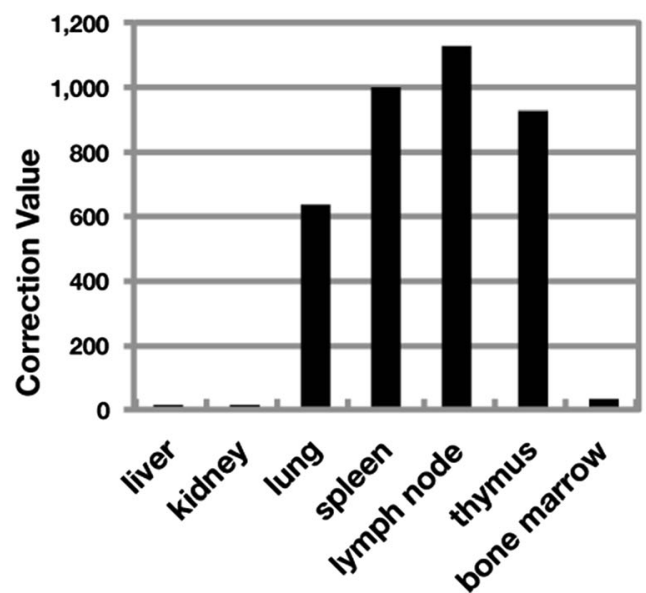

Fig. 7 Quantitative analysis of the expression of human transgenes in various tissues of TMC F8 mice. Each value was corrected by the level of mouse GAPDH (see Supplemental Fig. S2)

The $\mathrm{HAC}$ retention rate of tails was almost the same for the same individual at 6 weeks and 7 months of age (Table 2, Fig. 4a), and the HAC retention rate of the tail at 7 months was the same as that of the brain, heart, kidney, and liver at 7 months. The HAC retaining an early developmental cell proliferation stage in the TMC mouse may determine the consequent $\mathrm{HAC}$ retention rate in the tail, brain, heart, kidney, and liver. Thus, the HAC retention rate of the tail cells from young mice is a good and easy index for estimating the retention rate of these tissues. On the other hand, the retention rate of the bone marrow and spleen was relatively lower at 7 months of age. Whether the retention rate of the bone marrow and spleen will decrease with proliferating dependency requires further investigation.
Although the retention rates in the F1 mice harboring the DRA-HAC and the HLA-HAC were variable in individual mice, they were not significantly different on average ( 86.2 and $82.6 \%$, respectively). No significant decrease in stability was observed in the previous analysis after inserting three genes at different sites in the expression cassettes on the same HAC vector when evaluating cultured cells (data not shown). However, through the generations, significant differences were observed in the transmission rates between mice carrying the DRA-HAC and HLA-HAC. The DRA-HAC was generated by insertion of a 100-kb HLA-DRA BAC into one of four expression cassettes of the 25-4 HAC vector, whereas the HLA-HAC was generated by insertion of an additional 50kb HLA-DRB1 BAC into another expression cassette. This 
additional $\mathrm{BAC}$ insertion resulted in a significant decrease in the transmission rate through mouse generations and influenced the meiotic process in the germ line. The expression patterns and levels of HLA-DRA and HLA-DRB1 from the HAC vector were not significantly different and were restricted to immunocompetent mouse cells through the eight generations. Thus, the gene product from the additional HLADRB1 gene is not likely to be more harmful in the germ line. The additional BAC insertion at the separated expression cassette site on this circular HAC vector may have some more structural disadvantage through the meiotic process in the germ line. Indeed, we did not observe such stability differences between the DRA-HAC and the HLA-HAC through the $\mathrm{HAC}$ constructions in $\mathrm{CHO}$ cells and the HAC transfer until generation of chimeric mice. The additional BAC insertion may cause some structural change between the HAC centromere and the heterochromatin, both of which are responsible for the chromosome segregation and stability, and thought to be more dynamically changing through cellular senescence and between different types of cell lines (Maehara et al. 2010; Ohzeki et al. 2012). One possible explanation is that the differences of retention and transmission rates of the two HACs may be strongly influenced by epigenetic changes following mouse background conversion from BDF2 (ES) to B6 through the germ line. Although it is interesting to ask why an additional insertion decreases the stability of the circular HAC, we have already taken measures against it. Using the FLP-FRT system, HLA-DRA BAC and HLA-DRB1 BAC will be introduced into one expression cassette of the 25-4 HAC vector. We also developed two new site-specific recombination systems named VCre/VloxP and SCre/SloxP for genome engineering; the recognition sequences are different from the Cre recognition sequence (Suzuki and Nakayama 2011). These improvements enable us to introduce many BACs into one of chosen expression cassettes of the 25-4 HAC vector.

Although some instability was observed based on the manner of insertion, a de novo HAC vector carrying multiple genomic BACs was transmitted through at least eight generations, maintaining functional expression over seven generations, which is the minimum required for congenic breeding. We think that HAC vector technology opens up a new field that will offer advantages for generating transgenic animals that harbor multiple, large, single-copy DNA fragments and controlling the tissue-specific expression of transgenes.

Acknowledgments We would like to thank K. Sumi, Y. Hasegawa, and Y. Yamanaka for their technical assistance. This work was supported by a grant-in-aid for the Project for Developing Innovation Systems, Program for Fostering Regional Innovation (O.O.), from the Ministry of Education, Culture, Sports, Science and Technology of Japan and Core Research for Evolutional Science and Technology (CREST) JST (H.M.), and the Kazusa DNA Research Institute Foundation.
Open Access This article is distributed under the terms of the Creative Commons Attribution License which permits any use, distribution, and reproduction in any medium, provided the original author(s) and the source are credited.

\section{References}

Altmann DM, Takács K, Trowsdale J, Elliott JI (1993) Mouse mammary tumor virus-mediated T-cell receptor negative selection in HLADRA transgenic mice. Hum Immunol 37:149-156

Asami J, Inoue YU, Terakawa YW, Egusa SF, Inoue T (2011) Bacterial artificial chromosomes as analytical basis for gene transcriptional machineries. Transgenic Res 20:913-924

Chandler KJ, Chandler RL, Broeckelmann EM, Hou Y, Southard-Smith EM, Mortlock DP (2007) Relevance of BAC transgene copy number in mice: transgene copy number variation across multiple transgenic lines and correlations with transgene integrity and expression. Mamm Genome 18:693-708

Doherty AM, Fisher EM (2003) Microcell-mediated chromosome transfer (MMCT): small cells with huge potential. Mamm Genome 14: 583-592

Fukui Y, Esaki Y, Kimura A, Hirokawa K, Nishimura Y, Sasazuki T (1993) T-cell repertoire in a strain of transgenic C57BL/6 mice with the HLA-DRA gene on the X-chromosome. Immunogenetics 37: 204-211

Gong S, Zheng C, Doughty ML, Losos K, Didkovsky N, Schambra UB, Nowak NJ, Joyner A, Leblanc G, Hatten ME, Heintz N (2003) A gene expression atlas of the central nervous system based on bacterial artificial chromosomes. Nature 425:917-925

Harrington JJ, Van Bokkelen G, Mays RW, Gustashaw K, Willard HF (1997) Formation of de novo centromeres and construction of firstgeneration human artificial microchromosomes. Nat Genet 15:345355

Iida Y, Kim JH, Kazuki Y, Hoshiya H, Takiguchi M, Hayashi M, Erliandri I, Lee HS, Samoshkin A, Masumoto H, Earnshaw WC, Kouprina N, Larionov V, Oshimura M (2010) Human artificial chromosome with a conditional centromere for gene delivery and gene expression. DNA Res 17:293-301

Ikeno M, Masumoto H, Okazaki T (1994) Distribution of CENP-B boxes reflected in CREST centromere antigenic sites on long-range alphasatellite DNA arrays of human chromosome 21. Hum Mol Genet 3: $1245-1257$

Ikeno M, Grimes B, Okazaki T, Nakano M, Saitoh K, Hoshino H, McGill NI, Cooke H, Masumoto H (1998) Construction of YAC-based mammalian artificial chromosomes. Nat Biotechnol 16:431-439

Ikeno M, Suzuki N, Hasegawa Y, Okazaki T (2009) Manipulating transgenes using a chromosome vector. Nucleic Acids Res 37:e44

Ikeno M, Suzuki N, Kamiya M, Takahashi Y, Kudoh J, Okazaki T (2012) LINE1 family member is negative regulator of HLA-G expression. Nucleic Acids Res 40:10742-10752

Jux B, Staratschek-Jox A, Penninger JM, Schultze JL, Kolanus W (2013) Vav1 regulates MHCII expression in murine resting and activated B cells. Int Immunol 25:307-317

Kazuki Y, Oshimura M (2011) Human artificial chromosomes for gene delivery and the development of animal models. Mol Ther 19:15911601

Maehara K, Takahashi K, Saitoh S (2010) CENP-A reduction induces a $\mathrm{p} 53$-dependent cellular senescence response to protect cells from executing defective mitoses. Mol Cell Biol 30: 2090-2104

Miyamoto K, Suzuki N, Sakai K, Asakawa S, Okazaki T, Kudoh J, Ikeno M, Shimizu N (2014) A novel mouse model for Down syndrome that harbor a single copy of human artificial chromosome (HAC) 
carrying a limited number of genes from human chromosome 21 . Transgenic Res 23:317-329

Moro M, Cecconi V, Martinoli C, Dallegno E, Giabbai B, Degano M, Glaichenhaus N, Protti MP, Dellabona P, Casorati G (2005) Generation of functional HLA-DR*1101 tetramers receptive for loading with pathogen or tumour derived synthetic peptides. BMC Immunol 6:24

Nakayama M, Ohara O (2005) Improvement of recombination efficiency by mutation of Red proteins. Biotechniques 38:917-924

Ohtsuka M, Ogiwara S, Miura H, Mizutani A, Warita T, Sato M, Imai K, Hozumi K, Sato T, Tanaka M, Kimura M, Inoko H (2010) Pronuclear injection-based mouse targeted transgenesis for reproducible and highly efficient transgene expression. Nucleic Acids Res 38:e198

Ohtsuka M, Miura H, Sato M, Kimura M, Inoko H, Gurumurthy CB (2012) PITT: pronuclear injection-based targeted transgenesis, a reliable transgene expression method in mice. Exp Anim 61:489502

Ohzeki J, Bergmann JH, Kouprina N, Noskov VN, Nakano M, Kimura H, Earnshaw WC, Larionov V, Masumoto H (2012) Breaking the
HAC barrier: histone $\mathrm{H} 3 \mathrm{~K} 9$ acetyl/methyl balance regulates CENPA assembly. EMBO J 31:2391-2402

Pinkert CA, Widera G, Cowing C, Heber-Katz E, Palmiter RD, Flavell RA, Brinster RL (1985) Tissue-specific, inducible and functional expression of the $\mathrm{E}$ alpha $\mathrm{d} \mathrm{MHC}$ class II gene in transgenic mice. EMBO J 4:2225-2230

Suzuki E, Nakayama M (2011) VCre/VloxP and SCre/SloxP: new sitespecific recombination systems for genome engineering. Nucleic Acids Res 39:e49

Suzuki N, Nishii K, Okazaki T, Ikeno M (2006) Human artificial chromosomes constructed using the bottom-up strategy are stably maintained in mitosis and efficiently transmissible to progeny mice. J Biol Chem 281:26615-26623

Suzuki M, Takahashi T, Katano I, Ito R, Ito M, Harigae H, Ishii N, Sugamura K (2012) Induction of human humoral immune responses in a novel HLA-DR-expressing transgenic NOD/Shi-scid/ $\gamma$ cnull mouse. Int Immunol 24:243-252

Vintersten K, Testa G, Naumann R, Anastassiadis K, Stewart AF (2008) Bacterial artificial chromosome transgenesis through pronuclear injection of fertilized mouse oocytes. Methods Mol Biol 415:83-100 